\title{
Polarization insensitive blazed diffraction gratings
}

\author{
Nicolas Bonod \\ nicolas.bonod@fresnel.fr \\ Evgeny Popov \\ Stefan Enoch \\ Jérôme Néauport
}

\author{
Institut Fresnel, Université de Provence, CNRS UMR 6133, 13397 Marseille Cedex \\ Institut Fresnel, Université de Provence, CNRS UMR 6133, 13397 Marseille Cedex \\ Institut Fresnel, Université de Provence, CNRS UMR 6133, 13397 Marseille Cedex
}

Gratings insensitive to light polarization may be useful in many areas of optical engineering, particularly in light demultiplexing application. We study different kinds of binary gratings able to present reflected efficiencies higher than $98 \%$ in the -1 st order. These are metallic gratings, dielectric gratings deposited on a metallic substrate, total internal reflection gratings and gratings lying on a dielectric multilayer. We study each configuration with respect to manufacturing constraints and optical performances to conclude by providing the most suitable design. [DOI: 10.2971/jeos.2006.06029]

Keywords: Diffraction gratings, light demultiplexing, photonic crystals, polarization insensitive device

\section{Introduction}

Gratings present dispersive properties which are widely used in spectroscopy analysis, light demultiplexing and also in ultrashort pulse compression [1]. In many cases, they are used to disperse unpolarized incident light. Unfortunately, their diffractive properties are largely dependent on light polarization, therefore inducing undesired polarization dependent losses, undesirable for applications such as light demultiplexing [2]. This paper is devoted to the design of gratings able to diffract almost $100 \%$ of the incident light in a specific dispersive -1st order simultaneously for both transverse magnetic (TM) and electric (TE) polarization.

Three steps are necessary to diffract all the incident light in the -1 st order in reflection.

- The first one is to reflect the incident light. Metallic substrates, dielectric substrate used in total internal reflection, multilayer dielectric layers or photonic crystals can be used for this purpose.

- The second step is to etch a grating in the reflecting device. The ratio between the period and the incident wavelength determines the number of propagative orders. As the incident light energy is desired to be almost entirely diffracted into one specific order, the number of propagating orders must be minimal. When the angle of incidence is known, the number of propagating orders is only determined by the ratio between the wavelength $\lambda$ and the grating period $d$. The specular $(0)$ order is always propagative and does not present dispersive properties. The second propagating order is usually the
-1 st order. As a consequence, the grating period is determined to induce only two propagative orders. The exception to this rule concernes echelles grating with large period that blaze into an order with large number. They can be characterized with high values of efficiency in unpolarized light, however, the free spectral range in each diffraction order is quite limited.

- Finally, the last step consists of optimizing grating grooves profile to disperse all the incident light into the -1 st order.

In this paper, we will study different kinds of binary gratings which can diffract as much as possible the incident light in the -1 st order at the Littrow incidence. In the first section, we will consider two different metallic gratings. In the first case, the modulated layer lies in the metallic material, and in the second case, in a silica layer deposited onto the metallic substrate. In the second section, dielectric gratings based on total internal reflection phenomenon will be studied. These gratings are well known to diffract with a very good efficiency the incident light in the -1st order but to the best of our knowledge, no design has been proposed to make them insensitive to the incident polarization $[3,4]$. The third section is devoted to gratings etched on the surface of dielectric reflecting multilayers. We shall study both gratings recorded either on a dielectric mirror ( $\mathrm{HL})^{7} \mathrm{HL}$ made of $\mathrm{SiO}_{2}$ (for L) and $\mathrm{HfO}_{2}$ (for $\mathrm{H}$ ) or on a 2 dimensional photonic crystal made of macroporous silicon.

The wavelength is equal to $1.55 \mu \mathrm{m}$ for telecommunication ap- 
plications and the period $d$ is $1.33 \mu \mathrm{m}$, except in the second section where the total internal reflection condition imposes a lower period taken equal to $0.6 \mu \mathrm{m}$. The Littrow incidence in air is defined by $\sin \theta_{-1}=\lambda /(2 d)$, where $\theta_{-1}$ is the angle of refraction of the -1 st order, and is equal to $35.5 \mathrm{deg}$ [1]. It is well known that this incidence favourites the diffraction in the -1 st order [1]

Optimizations are made using a numerical code based on the differential method [5]. This method enables the rigorous resolution (to the numerical approximations) of Maxwell equations in periodic media.

\section{GRATINGS BASED ON METAL- LIC REFLECTOR}

\subsection{Metallic modulated area}

The most common metallic grating is a grating presenting a periodical modulation of the metallic surface. In practice, the relief is recorded in a photosensitive resin deposited onto a dielectric substrate (glass). After the photosensitive resin development, the modulated surface is coated by a metallic layer. The modulation can also be transferred directly into the dielectric substrate by ion etching technique and then coated by a metallic layer. In all these cases, the metallic layer is thick enough to be considered as the substrate and the device can be represented schematically as in Figure 1.

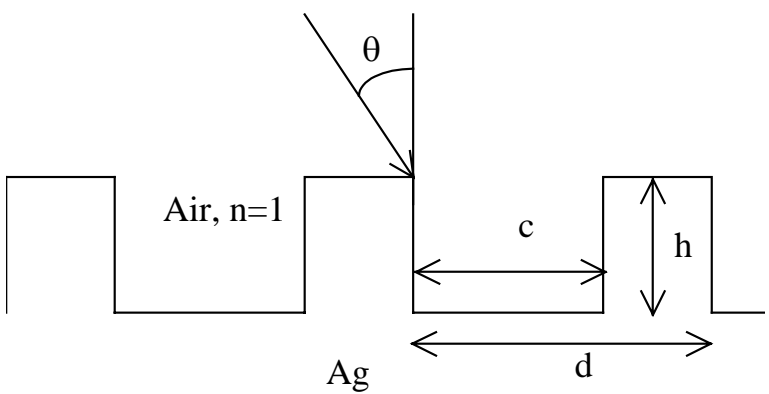

FIG. 1 Binary grating with groove height $\mathrm{h}$ and width $\mathrm{c}$. The grating is illuminated by a monochromatic plane wave of wavelength $\lambda \mathrm{g} 1.55 \mu \mathrm{m}$, with angle of incidence $\theta$. Grating period $d$ is equal to $1.33 \mu m$. Substrate is made of silver of optical index $n=0.322+i 10.9935$ and cladding is air.

The efficiencies in the -1-st reflected order are calculated in TE and TM polarization as a function of the groove height $h$ and width $c$, and the calculated efficiencies are given in Figure 2 in TE (Figure 2a) and TM (Figure 2b) polarization together with their mean values (Figure 2c). As it can be observed, the reflected efficiency in the -1st order depends highly on the polarization, and we can see in Figure 2c that the unpolarized efficiency does not reach $95 \%$. The use of the metal introduces too much losses thus limiting the efficiency to approximately $90 \%$ in both TE and TM polarization.

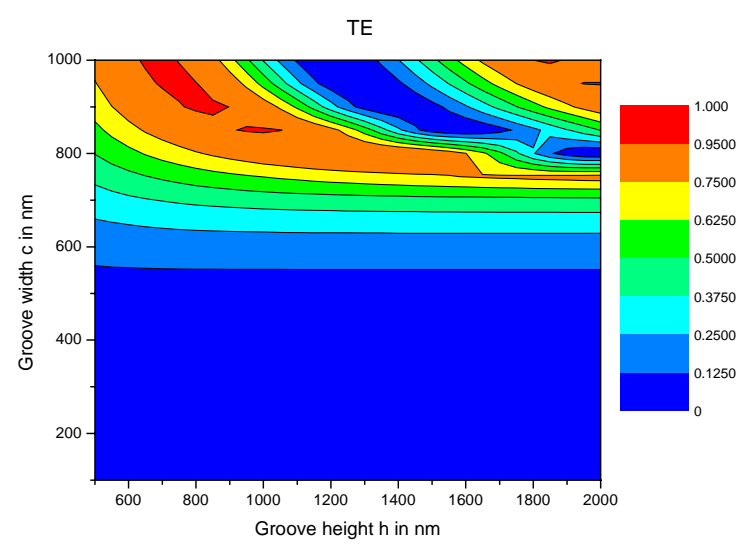

TM
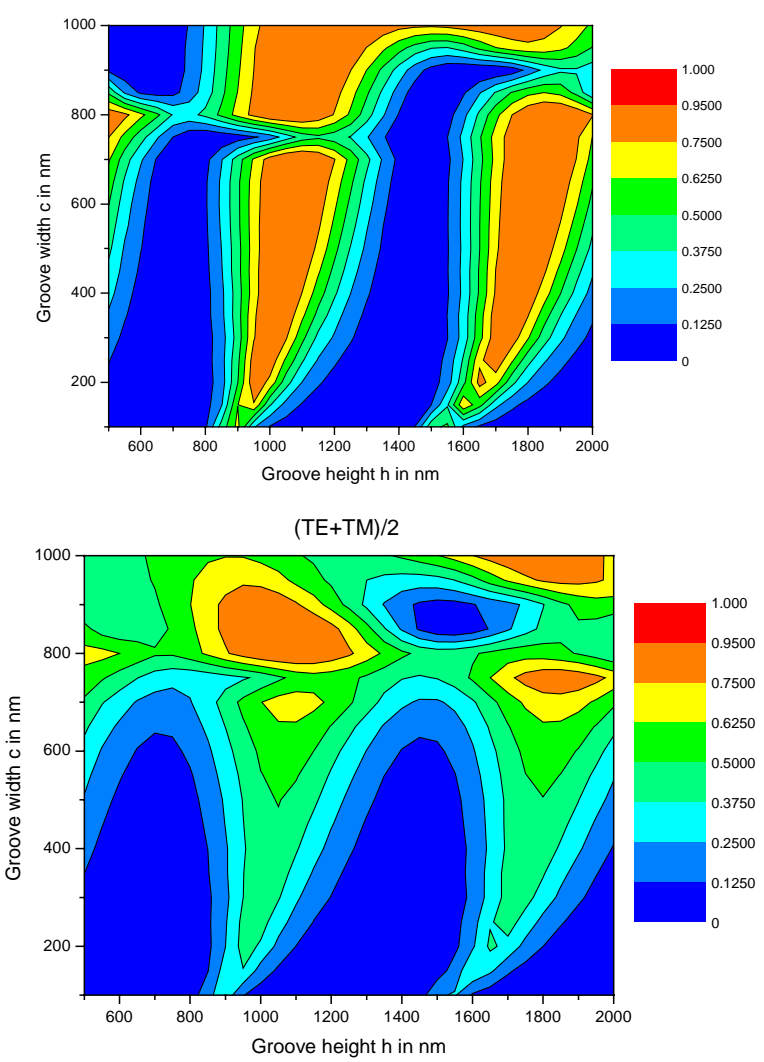

FIG. 2 Efficiencies in the -1st reflected order as a function of groove width $c$ and height h. (a): TE polarization, (b) TM polarization, (c) mean values of efficiencies for TE and TM polarization.

\subsection{Dielectric modulated area}

Let us now consider gratings made of a modulated dielectric layer deposited on a flat metallic substrate. The modulation is made into a usual dielectric material like silica deposited on a metallic reflective substrate. Silica presents a low refractive optical index allowing a larger tolerance to manufacturing imprecision with respect to the original profile. Gold is chosen since, compared to silver, it presents a much lower reflectivity at a wavelength $\lambda / 3$, used during the interference recording of the grating, which fact is of great importance in reducing the standing-wave effect inside the photoresist. The device under study is depicted in Figure 3. 


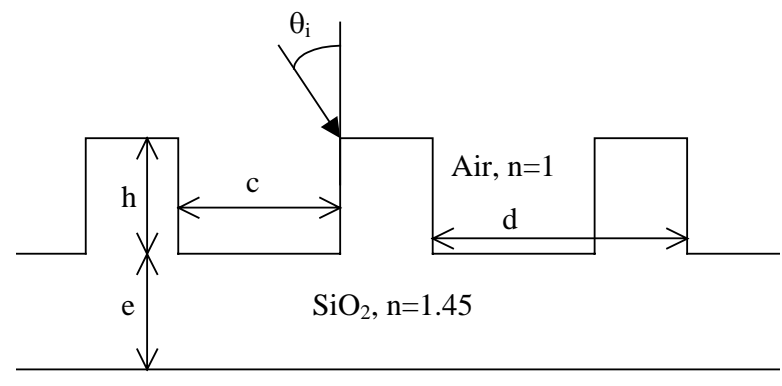

$\mathrm{Au}$

FIG. 3 A silica layer is deposited on a metallic substrate (gold) and etched periodically with a period $d$, a groove thickness $h$ and width $c$. The residual thickness of silica is e.

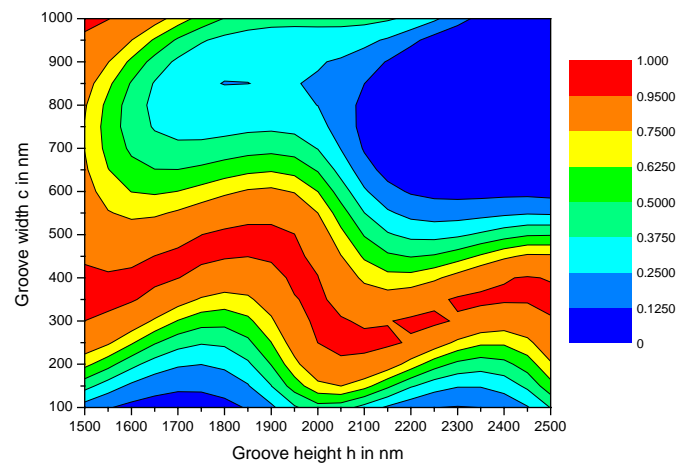

(a)

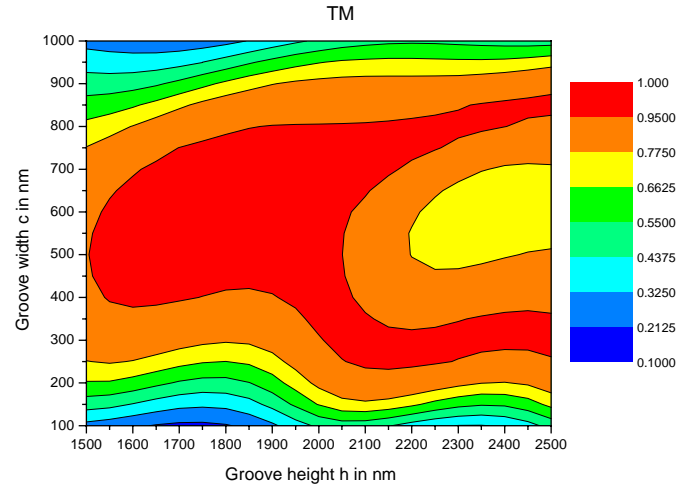

(b)

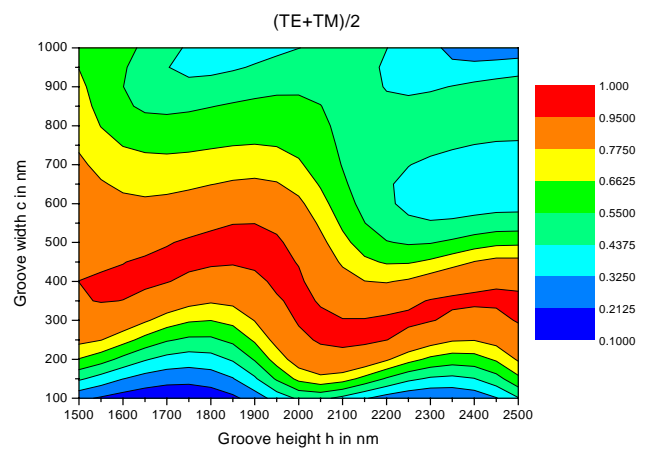

(c)

FIG. 4 Same as Figures 2, but for the grating presented in Figures 3.

It has been shown recently that such grating can diffract $99 \%$ of the incident light into the -1 st order [6] in TE polarization.
Guided modes in the silica layer are radiated by the modulated structure and constructive interferences between the propagated modes explain the almost perfectly diffraction [6].

The efficiency calculated as a function of the two parameters $h, c$ with $e=50 \mathrm{~nm}$ is shown in Figures 4 . The mean value of diffraction efficiencies in both polarizations is higher than $95 \%$. However, manufacturing of such profiles will be a hard task due to the large value of the groove height compared to the wavelength. Moreover, the groove width values, around $0.4 \mu \mathrm{m}$, which lead to the highest reflected efficiencies are small compared to the period $d(c / d=0.3$, and $h / \lambda>1)$. And indeed, conventional technologies used by grating manufacturers allow ratios $c / d$ around 0.3 only when the ratio $h / \lambda$ is largely smaller than 1 . Despite this technological limitation, such a grating present attractive properties.

\section{TOTAL INTERNAL REFLECTION G R A T I N G S}

In order to avoid the use of metal, undesirable for high energy applications, dielectric gratings based on the total internal reflection condition are now considered. The modulated area is illuminated from the material having higher optical index $n$ (Figure 5). It is now well known that this kind of grating can diffract almost all of the incident light in the -1 st order $[3,4]$. We first define the conditions on the grating period in order to satisfy the total internal reflection condition with two orders of propagations in Littrow incidence $\theta_{i}$. The total internal condition writes :

$$
\sin \theta_{i}>1 / n
$$

The Littrow incidence is defined by:

$$
\sin \theta_{-1}=-\lambda /(2 n d) .
$$

With the use of the two preceding conditions, one obtains:

$$
d<\lambda / 2
$$

The -1 st order is propagating if:

$$
d>\lambda / 2 n \text {. }
$$

Finally, the condition imposed on the grating period writes:

$$
\lambda / 2 n<d<\lambda / 2
$$

With $\lambda=1550 \mathrm{~nm}$ and $n=1.45$, one obtains:

$$
534 \mathrm{~nm}<d<775 \mathrm{~nm}
$$

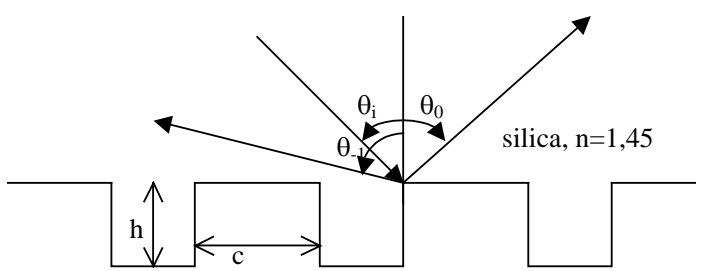

air

FIG. 5 Binary grating, working in total internal reflection and etched in silica illuminated by a monochromatic plane wave from the cladding made of silica, substrate is air. 
The period is taken equal to $d=600 \mathrm{~nm}$ and the Littrow incidence is then equal to $\sin \theta_{I}=\lambda / 2^{\text {nd }}=63^{\circ}$. Calculations of efficiencies, similar to the preceding section as a function of $d$ and $c$ lead to results shown in Figures 6.

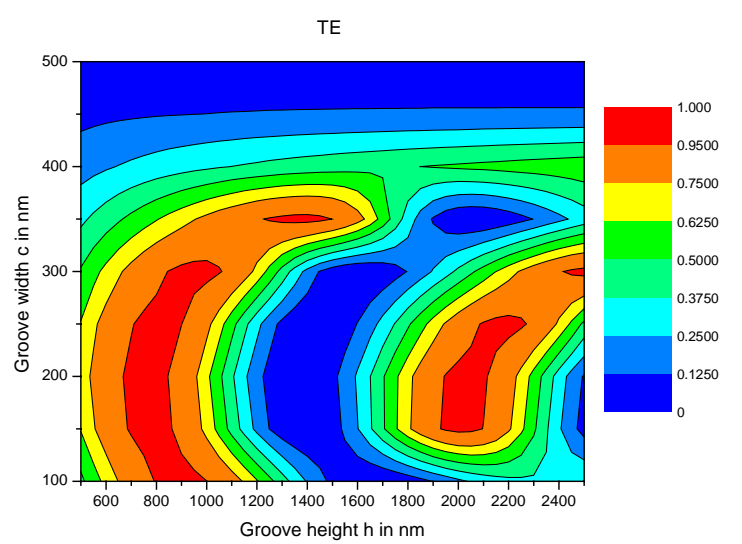

(a)



(b)



(c)

FIG. 6 Same as Figures 2 and 6, but for the grating presented in Figures 5

The average between diffraction efficiencies in TE and TM polarization reaches $98 \%$, unfortunately on a very narrow area. The optical properties of this grating appear to be highly dependent on the groove geometry, which is a severe technological limitation.

\section{M ULTILAYER DIELECTRIC GRAT- I N G S}

The most common dielectric reflecting device is the well known dielectric Bragg mirror. A stack of alternatively high and low optical index material is deposited onto a dielectric substrate. The optical thickness of the layers is equal to $\lambda / 4 n_{i} \cos \theta_{i}$ where $n_{i}$ is the optical index of the $\mathrm{i}$-th layer and $\theta_{i}$ is the angle of refraction of the plane wave in this layer. The stack under study contains 7 bilayers of high and low optical index plus a layer of high optical index on the top, and is denoted as $(\mathrm{HL})^{7} \mathrm{H}$. A thicker layer of low-index material is then deposited to be etched periodically. The residual thickness of the low-index is denoted as $e$ (Figure 7). This kind of grating was designed in the middle of the 90's to increase the laser damage threshold of gratings used for ultrashort laser pulses compression $[7,8]$.

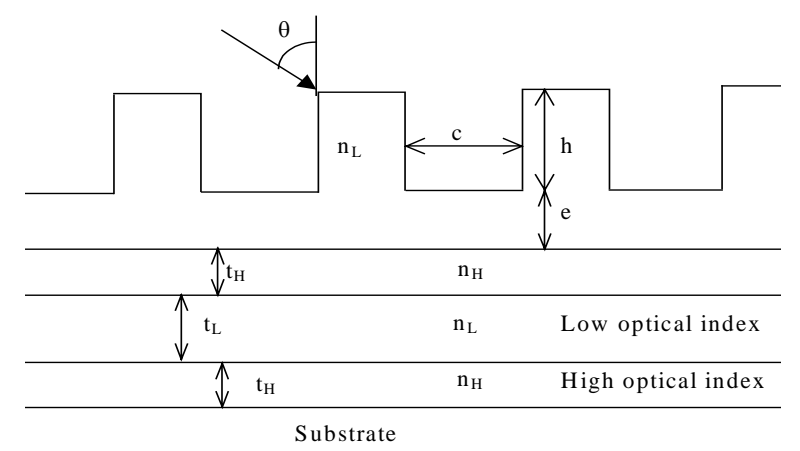

FIG. 7 Multilayer dielectric grating $(\mathrm{HL})^{7} \mathrm{H}$. Substrate is pyrex, high optical index material is $\mathrm{HfO}_{2}$, and low optical index material is $\mathrm{SiO}_{2}$.

At first, we study the reflectivity of the mirror $(\mathrm{HL})^{7} \mathrm{H}$ as a function of the angle of incidence $\theta$ or the both polarizations (Figure 8).

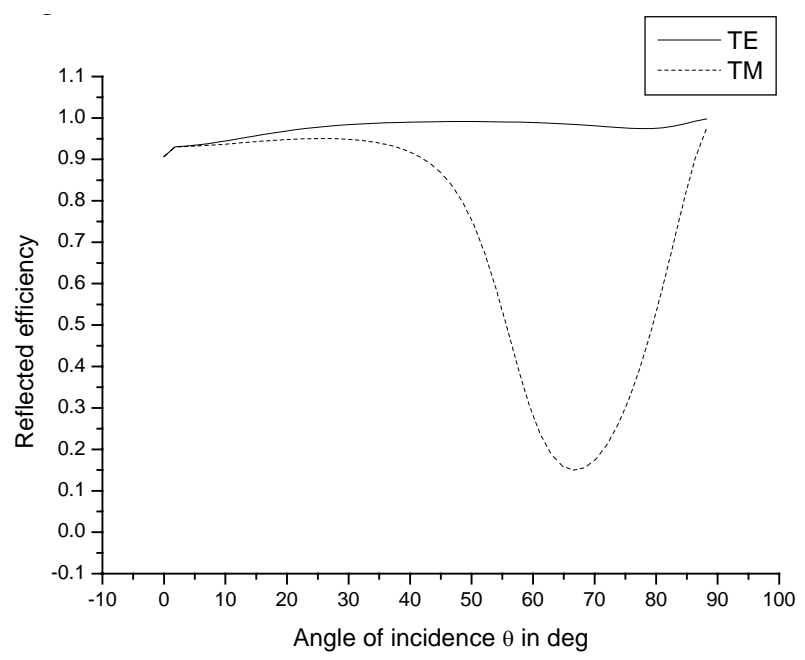

FIC. 8 Reflectivity of the dielectric mirror $(\mathrm{HL})^{7} \mathrm{H} . n_{H}=1.9$ and $n_{B}=1.46$.

As the reflectivity is much better in TE polarization than in TM polarization, we expect to observe diffracted efficiencies largely dependent on the polarization. And indeed, the aver- 
aged reflectivity does not reach $90 \%$, due to the insufficient reflectivity of the stack (Figure 9) in TM polarization.

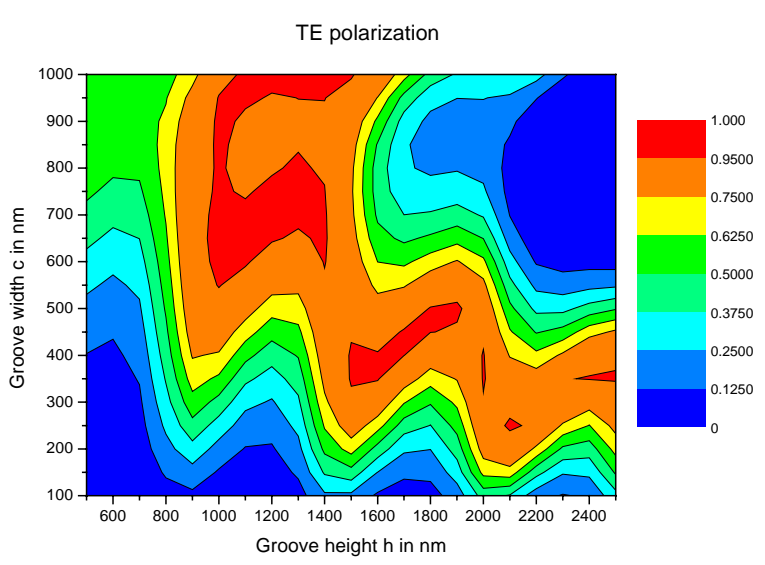

(a)



(b)

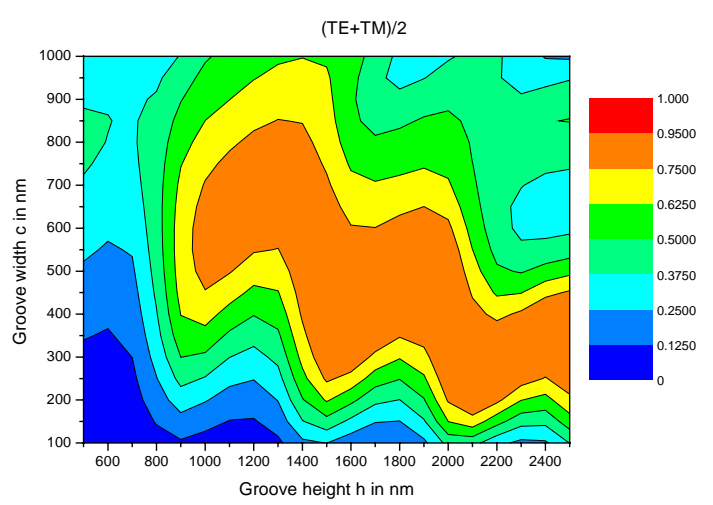

(c)

FIG. 9 Efficiency of the -1 st order in reflection, averaged in TE and TM polarizations with $e=50 \mathrm{~nm}$.

To eliminate this difficulty, we can increase the number of bilayers, but mechanical stress grows resulting in crackings inside the stack or deformation of the wavefront depending on the coating process.

Another possibility is to increase the optical contrast between the layers. Let us increase the value of the high optical index up to 3.4 , which corresponds to the silicon at $\lambda=1.55 \mu \mathrm{m}$, keeping the same number of bilayers. We can observe in Figure 10 that the increase of the optical contrast leads to a better reflectivity of the dielectric mirror, even if the reflectivity in TM polarization is still lower, especially at grazing incidences.

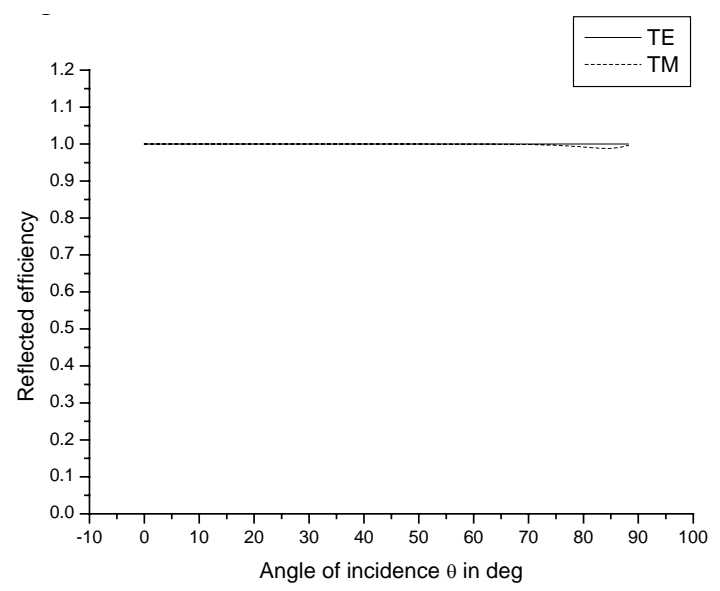

FIG. 10 Reflectivity of the dielectric mirror $(\mathrm{HL})^{7} \mathrm{H} . n_{H}=3.4$ and $n_{L}=1.46$.

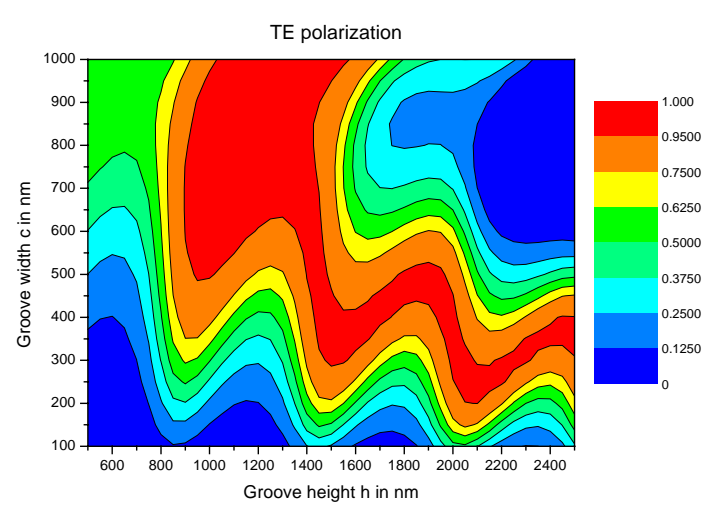

(a)



(b)

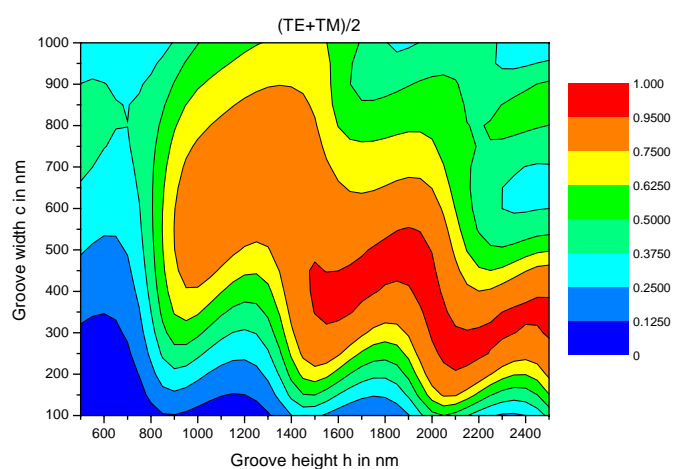

(c)

FIC. 11 Efficiency in the -1st reflected order averaged between the both polarizations of the grating recorded on the stack $(\mathrm{HL})^{7} \mathrm{HL}$ with $n_{H}=3.4$ and $n_{L}=1.46, e=50 \mathrm{~nm}$. 
The same calculations of efficiency as a function of $h$ and $c$ result in plots shown in Figure 11. It is well shown in Figure $11 c$ that diffraction efficiency in the -1st order in unpolarized light is higher than $97 \%$ over a large area, i.e. with high tolerances to manufacturing defaults. As a consequence, a grating based on a high-contrast dielectric mirrors appears to be well adapted for the design of polarization insensitive gratings.

\section{PHOTONIC CRYSTAL DIFFRAC- TIONGRATING}

Nowadays, the progress of technology has made possible the realization of $2 \mathrm{D}$ photonic crystals. They appear as new opportunities to design reflection gratings. We have chosen a photonic crystal consisting of square holes in a silicon matrix [9]. The geometry is invariant in the $\mathrm{z}$ direction, periodic and infinite in the $x$ direction (Figure 12).

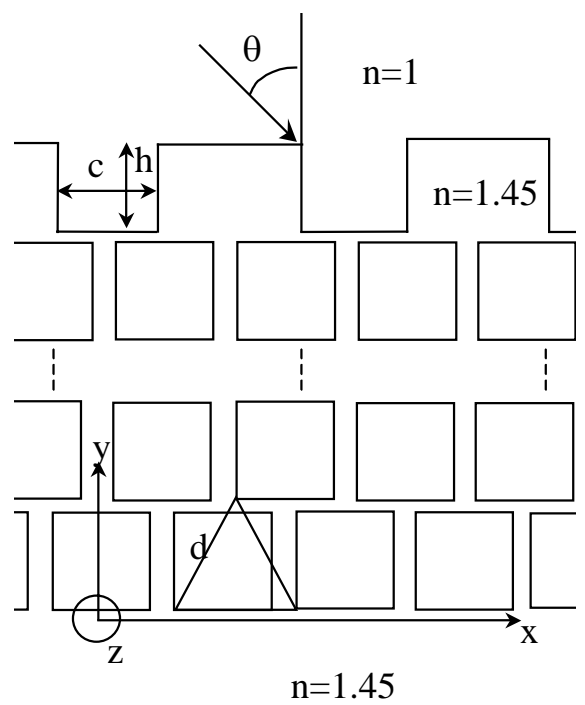

(a)

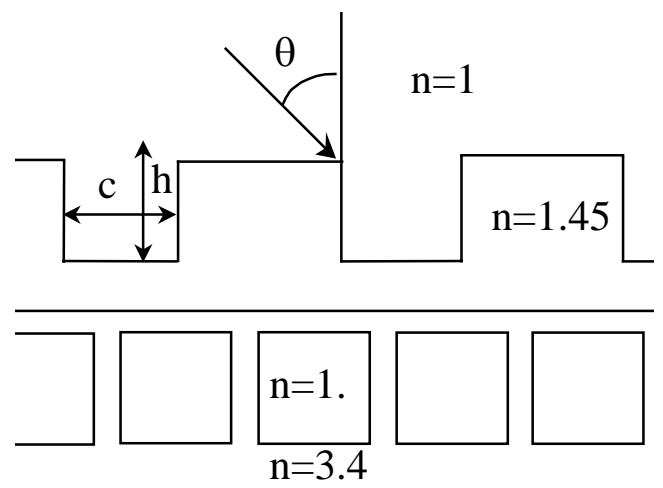

(b)

FIG. 12 (a) A 2 dimensional photonic crystal of triangular lattice, finite in the $y$ direction, infinite and periodic in the $x$ direction, and invariant in the $z$ direction. It is made of 8 periodically modulated layers of period $d=0.66 \mu \mathrm{m}$. Square holes made in silicon have a length equal to $0.8 d$. (b) A grating is etched in a silica layer deposited on the photonic crystal. The grating period is twice bigger than the square holes period $d$.
The period $d$ of square holes $(0.66 \mu \mathrm{m})$ equal to the half of the grating period $(1.33 \mu \mathrm{m})$, is small enough to prevent propagation of dispersive orders. In the y direction, photonic crystal is made of 8 layers of periodical square holes of length equal to $0.8 d$. Substrate is pyrex, and cladding is air. The residual thickness of silicon above and below the square lattice is equal to the half of the thickness of the layer positioned between the two layers of periodic square holes, i.e, equal to $d(\sin (\pi / 3)-0.8) / 2$. This photonic crystal presents photonic band gaps common to both TE and TM polarizations, centred around the wavelength of study $1.55 \mu \mathrm{m}$.

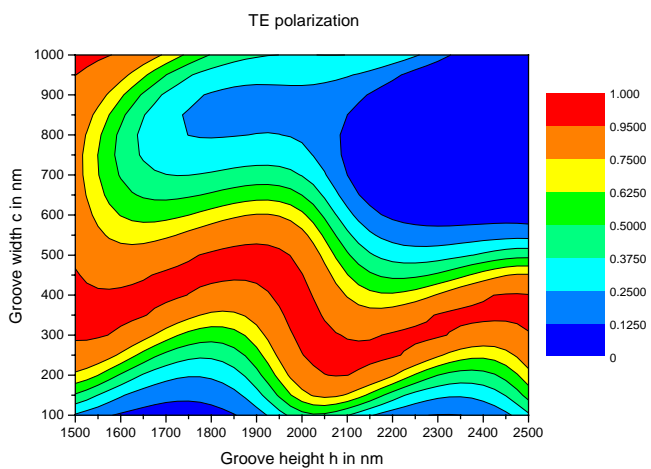

(a)

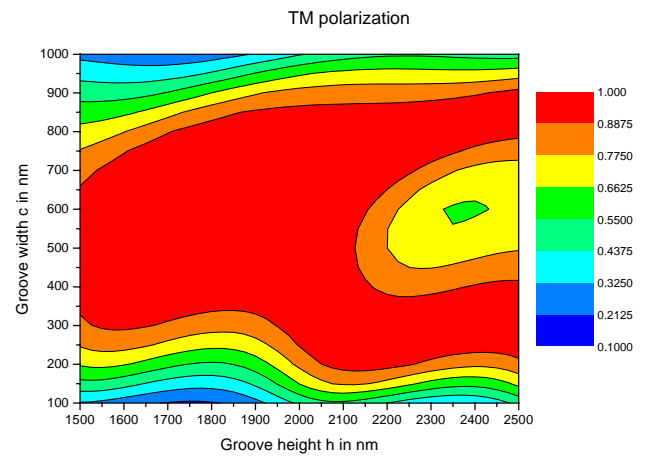

(b)

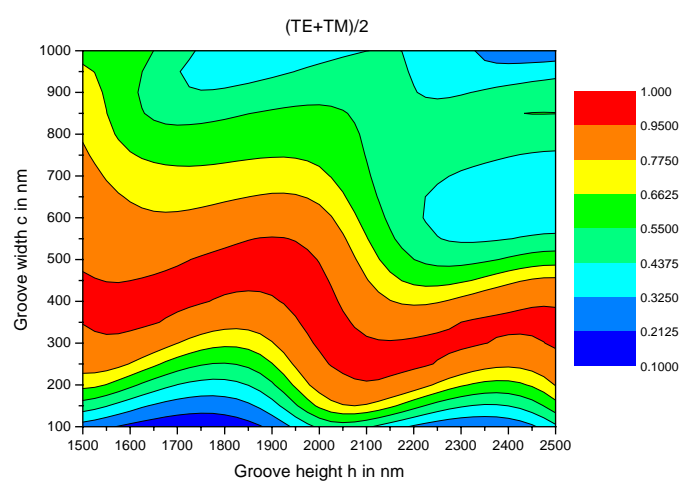

(c)

FIG. 13 Average of diffraction efficiencies in TE and TM polarization as a function of groove height and width for the grating made inside a silica layer deposited on the top the photonic crystal at Littrow incidence with $\lambda=1.55 \mu \mathrm{m}$.

It can be observed that the reflectivity is almost perfect for both polarizations over a broad range of incidence. Contrary to the dielectric mirror studied in the preceding section, the reflectivity of the photonic crystal is better in TM polarization than in TE polarization. 
To observe the propagation of the -1st dispersive order, a grating with period $2 \mathrm{~d}$ is etched in a silica layer deposited on the top of the photonic crystal, with a residual thickness of silica denoted $e$ (Figure 13). Such a device can be called photonic crystal diffraction grating $[10,11]$. To rigorously model such a device, a numerical code has been developed to solve Maxwell equations in periodical devices presenting 2 periods, one being twice bigger than the other.

Calculations of efficiencies as a function of groove width $c$ and height $\mathrm{h}$ similar to preceding sections is undertaken. Mean efficiency higher than $97 \%$ can be observed over a large area of groove parameters (Figure 13).



(a)

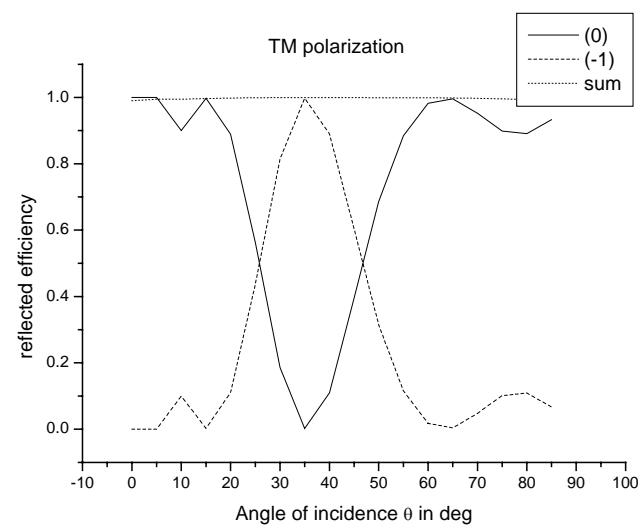

(b) ciencies is directly related to the angular tolerance of the photonic crystal, and as expected, we can observe in Figures 14 the perfect reflection of the device over the range of incidence and conclude that the angular tolerance of the photonic crystal diffraction grating is imposed by the angular tolerance of the grating.

\section{C O N C L U SIO N}

We discuss different possibilities to design binary diffraction gratings able to diffract almost all the incident light (98\%) in the -1 st order in reflection, simultaneously for both fundamental polarizations. These gratings may considerably reduce the polarization dependent losses in telecommunication applications. We have studied and compared several types of gratings based on: metallic mirror, total internal reflection phenomenon and photonic crystal band gaps. Diffraction efficiencies of gratings with a metallic modulated surface are limited by losses induced by the metal, despite of the use of a highly conducting metal (silver). Much better performances are obtained when the modulated area lies inside a dielectric layer made of silica, whatever the reflecting device. Mean efficiencies averaged on both polarizations can reach values as high as $98 \%$. The criterion used to select the best design is the stability of optical properties with respect to variations of groove geometry induced by technological processes. Large optical tolerance are obtained in Figures $(4,6,13)$, in which the domain of high reflected efficiencies ( $>95 \%$ ) covers a large area of groove parameters. The largest area is obtained in case of diffraction grating recorder on a 2D photonic crystal. This device appears to have high tolerances with respect to groove profile variations and provides polarization insensitive properties around values of $c$ slightly lower than $d / 2$ and $h$ slightly higher than $\lambda$, which are easily accessible with actual technology. Photonic crystals are able to reflect light over a large range of angle of incidence. In order to know if this property may be useful for the design of grating poorly sensitive to incidence, reflected efficiencies of both propagating orders are plotted as a function of the angle of incidence. It is clearly shown that the angular tolerance is only determined by the grating's one since the sum of the both orders is almost equal to 1 over the whole range of incidence.

\section{References}

[1] G. Loewen and E. Popov, Diffraction Gratings and Applications (Marcel Dekker, Inc, 1997).

[2] E. Popov, J. Hoose, B. Frankel, C. Keast, M. Fritze, T. Y. Fan, D. Yost, and S. Rabe, "Low polarization dependent diffraction grating for wavelength demultiplexing" Opt. Express 12, 269-275 (2004).

[3] E. Popov, L. Mashev, and D. Maystre, "Back-side diffraction by relief gratings" Opt. Commun. 65, 97-100 (1988).

[4] J. R. Marciante and D. H. Raguin, “High efficiency, high dispersion diffraction gratings based on total internal reflection" Opt. Lett. 29, 542-544 (2004).

[5] M. Nevière, E. Popov, Light propagation in periodic medias; differential theory and design, (Marcel Dekker, New York, Basel, Honk Kong, 2003). TM (Figure 14b) polarizations. The sum of the reflected effi(0th and -1st) as a function of $\theta$, for both TE (Figure 14a) and

ing and the photonic crystal are compared. The reflected efficiencies are calculated as a function of the incident angle $\theta$ with profile dimensions $h=1850 \mathrm{~nm}$ and $c=450 \mathrm{~nm}$, a couple which leads to the highest mean efficiency. We present in Figures 14 the -1 st order and the sum of the both reflected orders (19. 
[6] N. Destouches, A. Tishchenko, J. Pommier, S. Reynaud, 0. Parriaux, S. Tonchev, M. Ahmed, " $99 \%$ efficiency measured in the -1 " ${ }^{\text {st }}$ order of a resonant grating" Opt. Express 13, 3230-3235 (2005).

[7] M. D. Perry, R. D. Boyd, J. A. Britten, B. W. Shore, C. Shannon and L. Li, "High efficiency multilayer dielectric diffraction gratings" 0 pt. Lett. 20, 940-942 (1995).

[8] N. Bonod, J. Néauport, "Optical performances and laser induced damage threshold improvement of diffraction gratings used as compressor in ultra high intensity laser" Opt. Commun. 260, 649655 (2006).

[9] K. Sakoda, Optical Properties of Photonic Crystals, (Springer, Berlin, 2001).

[10] E. Popov, B. Bozhkov, and M. Neviere, "Almost perfect blazing by photonic crystal rods gratings" Apll. Opt. 40, 2417-2422 (2001).

[11] D. Maystre, "Photonic crystal diffraction grating" Opt. Express 8, 209-216 (2001) 